\title{
A Fully Probabilistic Decentralised Control Design for Complex Stochastic Systems*
}

\author{
Randa Herzallah ${ }^{1}$
}

\begin{abstract}
Computational and communication complexity call for robustness of controlled systems as well as for distributed control. The proposed technical solutions in this paper are bottom up solutions where simple controllers are designed to care about individual nodes either completely independently or within various structures like cascade control. Cheap computational resources allow now the improvement of the overall behaviour of the network of such controlled loops by allowing the individual "nodes" to share information with their neighbours without aiming at hopeless global solution. The current paper inspects this proposed method on a linearised version of coupled map lattice with spatiotemporal chaos yielding close to linear quadratic design which gives insight into possible behaviours of such networks.
\end{abstract}

\section{INTRODUCTION}

Many systems in nature, technology and society can be regarded as complex networks which consist of a large number of elements interacting with each other. The scale and nature of such systems make them pervasive, inherently nonlinear, coupled and operating under high levels of uncertainty. These attributes make the analysis, estimation and especially control of such systems a significant challenge which has yet to be adequately addressed. Several estimation and control methods have been proposed in the literature, including pinning control [1], multi-agent control [2], partial probabilistic control, decentralised [3] and distributed control [4]. The majority of the developed research in distributed control systems assume access to the global information of the system, follow deterministic architecture, and do not allow intelligent and adaptive decisions to be taken, as emphasised by a recent guest editorial paper [4].

This paper takes a different approach: the computational and communication complexities in large scale systems are to be addressed via distributed stochastic control where noise and uncertainties are contained using probabilistic approaches. In many such systems, although there may be a large number of components, these components are coupled, thus reducing the degrees of freedom, and introducing constraints. A complex performance function or set of functions may decompose so that the control of large networks can be developed in a bottom-up way in which simple controllers focus on individual nodes either completely independently or within various architectures such as cascade control. Access to cheap computational resources now allows improvement

\footnotetext{
*The work was funded by a Leverhulme Trust Research Project Grant number RPG-2017-337

${ }^{1}$ Randa Herzallah is with Systems Analytics Research Institute, School of Engineering and Applied Science, Aston University, B4 7ET Birmingham, United Kingdom r.herzallaheaston.ac.uk
}

of the overall behaviour of the network of such controlled architectures by allowing the individual nodes to share information with their neighbours without aiming for unattainable global solutions.

Message-passing is one of the new classes of decentralised methods for managing systems with large ensembles of interconnected nodes [5], [6] where information is retrieved and disseminated in a consistent probabilistic fashion. The approach has emerged independently in a number of field, including: communications theory [7], artificial intelligence [8], and statistical physics [9]. However, the techniques and their potential generalisations are modestly known within the control community. An objective of this paper is to generalise these methods in the context of decentralised probabilistic control, thus extending the methods from a passive to an active domain. This approach of a collection of decentralised probabilistic controllers passing information through probabilistic 'messages', can decompose the control of the large-scale networks into a collection of smaller control problems, one for each connected subnetwork in the system. These sub-control problems are treated independently and can therefore be analysed and implemented individually.

To obtain robust, reliable and efficient control inputs, the performance objective function is required to be a probabilistic function. Although some research has considered probabilistically controlling complex dynamical systems, the implementation of probabilistic performance functions has not been researched extensively despite its clear significant engineering importance. We have developed and extended fully probabilistic design methods (FPD) [10], [11], in which the optimal controller is the minimiser of the KulbackLeibler divergence, for single dynamical systems. This fully probabilistic design method will underpin this paper for distributed control systems with multiple subsystems.

This paper therefore proposes a novel way of reducing the amount of computation required in the optimisation, control, and estimation of complex networked systems. Our hypothesis is that in successful distributed estimation and control systems, the global behavioural performance of complex systems should be an emergent property. According to this hypothesis, the emergence of a complex property (global behavioural performance) can be realised by understanding simpler subsystems behaviour (the localised behaviour of controlled subsystems). Thus, to realise our hypothesis we aim to design and develop sparse or distributed control methods for the individual subsystems so that the desired emergent global behavioural performance of the system is 
achieved. Nodes exchange information or messages with their neighbours according to the topology of the graph. The messages are then updated iteratively in a probabilistic framework, and are used to estimate the dynamics of decision variables in the network.

To summarize, this paper aims at developing and understanding the properties of the proposed probabilistic distributed controllers and the information sharing between these controllers. For that purpose the proposed method is demonstrated and inspected in this paper on a simple linear quadratic example. The relationship between the proposed distributed probabilistic control methodology in this paper and the standard pinning control techniques will also be developed and the comparison between the two approaches will be established. Simulation results proof the efficacy of the proposed distributed controllers in achieving the optimal performance of the controlled system with less computational and design efforts when compared to pinning control method.

\section{Problem Formulation}

The controlled multivariate plant has the measurable state $x_{t+1} \in R^{n}$ and it is controlled by the (system) input $u_{t} \in$ $\mathrm{R}^{\mathrm{m}}$. The plant exhibits random behaviour, therefore, only the conditional probability density functions (pdfs) of the future state values can be specified at each instant of time as follows

$$
s\left(x_{t+1} \mid u_{t}, x_{t}\right) \text {. }
$$

Within the fully probabilistic design approach, this plant is controlled by randomised controllers described by conditional pdfs

$$
c\left(u_{t} \mid x_{t}\right)
$$

that minimizes the Kulback-Leibler divergence (KLD) between the actual joint pdf of the closed loop behavior of the plant, $f\left(x_{t+1}, u_{t} \mid x_{t}\right)$ and a predefined desired pdf, $I_{f}\left(x_{t+1}, u_{t} \mid x_{t}\right)$,

$$
\begin{aligned}
& \mathcal{D}\left(f \|{ }_{f}\right) \equiv \sum_{t=1}^{H} \int f\left(x_{t+1}, u_{t} \mid x_{t}\right) \ln \left(\frac{f\left(x_{t+1}, u_{t} \mid x_{t}\right)}{I_{f}\left(x_{t+1}, u_{t} \mid x_{t}\right)}\right) \\
& d x_{t+1} d u_{t} .
\end{aligned}
$$

This design method was originally presented in [10] where the probabilistic controller is obtained such that it minimizes the Kullback-Leibler divergence distance defined in (3). The minimum cost function resulting from minimization of (3) with respect to admissible control sequence $u_{t}, t \in$ $\{1, \ldots, H\}$, generated by the sequence of the randomised controllers 2 with $\mathrm{H}$ being the control horizon, is then shown to be given by the following recurrence equation [11],

$$
\begin{aligned}
& -\ln \left(\gamma\left(x_{t}\right)\right)=\min _{c\left(u_{t} \mid x_{t}\right)} \int s\left(x_{t+1} \mid u_{t}, x_{t}\right) c\left(u_{t} \mid x_{t}\right) \\
& \times[\underbrace{\ln \left(\frac{s\left(x_{t+1} \mid u_{t}, x_{t}\right) c\left(u_{t} \mid x_{t}\right)}{I_{s}\left(x_{t+1} \mid u_{t}, x_{t}\right) I_{c} c\left(u_{t} \mid x_{t}\right)}\right)}_{\equiv \text { partial cost } \Longrightarrow U\left(x_{t+1}, u_{t}\right)}-\underbrace{\ln \left(\gamma\left(x_{t+1}\right)\right)}_{\text {optimal cost-to-go }}] \\
& d\left(x_{t+1}, u_{t}\right),
\end{aligned}
$$

where $-\ln \left(\gamma\left(x_{t}\right)\right)$ is the expected minimum cost-to-go function and

$$
f\left(x_{t+1}, u_{t} \mid x_{t}\right)=s\left(x_{t+1} \mid u_{t}, x_{t}\right) c\left(u_{t} \mid x_{t}\right),
$$

is the factorisation of the actual joint pdf by the chain rule [12], which represents the most complete probabilistic description of the closed loop system. Here the pdf $s\left(x_{t+1} \mid u_{t}, x_{t}\right)$ describes the dynamics of the observed state vector $x_{t+1}$. Similarly

$$
{ }^{\mathrm{I}} \mathrm{f}\left(x_{\mathrm{t}+1}, \mathrm{u}_{\mathrm{t}} \mid x_{\mathrm{t}}\right)={ }^{\mathrm{I}} \mathrm{s}\left(\mathrm{x}_{\mathrm{t}+1} \mid \mathrm{u}_{\mathrm{t}}, \mathrm{x}_{\mathrm{t}}\right){ }^{\mathrm{I}} \mathrm{c}\left(\mathrm{u}_{\mathrm{t}} \mid \mathrm{x}_{\mathrm{t}}\right),
$$

is the factorisation of the ideal joint pdf of the closed loop system and ${ }^{I_{s}}\left(x_{t+1} \mid u_{t}, x_{t}\right)$ and ${ }^{I} c\left(u_{t} \mid x_{t}\right)$ represent the pdfs of the desired dynamics of the observed state vector and ideal controller respectively. The pdf of optimal controller, $c^{*}\left(u_{t} \mid x_{t}\right)$, minimizing the cost-to-go function (4) is shown [11], [13] to be determined by the following recursion

$$
\begin{aligned}
& c^{*}\left(u_{t} \mid x_{t}\right)=\frac{{ }^{I} c\left(u_{t} \mid x_{t}\right) \exp \left[-\beta\left(u_{t}, x_{t}\right)\right]}{\gamma\left(x_{t}\right)} \\
& \gamma\left(x_{t}\right)=\int I^{I} c\left(u_{t} \mid x_{t}\right) \exp \left[-\beta\left(u_{t}, x_{t}\right)\right] d u_{t} \\
& \beta\left(u_{t}, x_{t}\right)=\int s\left(x_{t+1} \mid u_{t}, x_{t}\right)\left[\ln \frac{s\left(x_{t+1} \mid u_{t}, x_{t}\right)}{I_{s}\left(x_{t+1} \mid u_{t}, x_{t}\right)}\right. \\
& \left.-\ln \left(\gamma\left(x_{t+1}\right)\right)\right] d x_{t+1} .
\end{aligned}
$$

However in large scale systems the above methodology for designing probabilistic controllers can become computationally very intensive. Therefore, the above FPD control method has been extended in [14] to control complex stochastic dynamical networks via pinning control. The optimized probabilistic pinning control laws have the same form as specified by equation 7 , but control inputs are added only to a fraction of nodes in the network. Therefor FPD control and FPD pinning control will be used interchangeably throughout this paper to mean the same thing.

This paper takes a different approach: the computational and communication complexities in large scale systems are to be addressed via distributed stochastic control where noise and uncertainties are contained using fully probabilistic approaches. Within this formulation, we decompose the complex system into $|\mathbf{I}|$ subsystems each controlled by a randomised controller described by pdfs $c\left(u_{t ; i} \mid w_{t ; i}\right)$, where $i \in \mathbf{I} \equiv\{1, \ldots,|\mathbf{I}|\}$. The $i$ th controller controls the outputs of the ith subsystem, $x_{t+1 ; i}$, which are also driven by part of the observed multivariate state values of their neighbours, $y_{t ; i}$ received through message passing. Hence, the pdfs of the ith is subsystem is defined as follows,

$$
\begin{aligned}
& s\left(x_{t+1 ; i}, y_{t+1 ; i} \mid u_{t ; i}, w_{t ; i}\right)=s\left(x_{t+1 ; i} \mid u_{t ; i}, w_{t ; i}\right) \\
& \times s\left(y_{t+1 ; i} \mid y_{t ; i}\right) \\
& w_{t ; i}=\left[x_{t ; i}, y_{t ; i}\right], \quad w_{0 ; i} \text { given }
\end{aligned}
$$

The performance function 4 is then used to design the individual controllers described above. For this purpose, the 
FPD expresses the control aim via ideal closed loop model given by a pdf

$$
\begin{aligned}
& { }^{I} s\left(x_{t+1 ; i}, y_{t+1 ; i} \mid u_{t ; i}, w_{t ; i}\right)={ }^{I} s\left(x_{t+1 ; i} \mid u_{t ; i}, w_{t ; i}\right) \\
& \times s\left(y_{t+1 ; i} \mid y_{t ; i}\right),
\end{aligned}
$$

which takes the pdf modelling the external variables to be equal to the ideal (desirable) pdf of external variables, thus not influencing their behaviours.

The specialization of the ith controller with the assumed model of subsystem states 8 , the ideal distribution of the ith subsystem 9, and the ideal distribution of the ith controller ${ }^{\mathrm{I}} \mathrm{c}\left(\mathrm{u}_{\mathrm{t} ; \mathrm{i}} \mid \boldsymbol{w}_{\mathrm{t} ; \mathrm{i}}\right)$ yields optimal solution specified in the following proposition.

Proposition 1: The pdf of the ith optimal controller minimizing the cost-to-go function 4 is given by

$$
\begin{aligned}
& c^{*}\left(u_{t ; i} \mid w_{t ; i}\right)=\frac{I_{c}\left(u_{t ; i} \mid w_{t ; i}\right) \exp \left[-\beta\left(u_{t ; i}, w_{t ; i}\right)\right]}{\gamma\left(w_{t ; i}\right)} \\
& \gamma\left(w_{t ; i}\right)=\int I c\left(u_{t, i} \mid w_{t ; i}\right) \exp \left[-\beta\left(u_{t, i}, w_{t ; i}\right)\right] d u_{t, i} \\
& \beta\left(u_{t, i}, w_{t ; i}\right)=\int s\left(x_{t+1, i} \mid u_{t, i}, w_{t ; i}\right) \\
& {\left[\ln \frac{s\left(x_{t+1, i} \mid u_{t, i}, w_{t ; i}\right)}{I_{s}\left(x_{t+1, i} \mid u_{t, i}, w_{t ; i}\right)}-\ln \left(\tilde{\gamma}\left(x_{t+1, i}, y_{t ; i}\right)\right)\right] d x_{t+1, i}} \\
& -\ln \left(\tilde{\gamma}\left(x_{t+1, i}, y_{t ; i}\right)\right)=\int s\left(y_{t+1, i} \mid y_{t ; i}\right) \\
& \ln \gamma\left(w_{t+1}\right) d y_{t+1, i}
\end{aligned}
$$

Proof: The result is implied by the following sequence of equalities in which one cost-to-go function 4 is dedicated to obtain the optimal control solution for each of the ith controller. We also use Fubini theorem on multiple integration [15], marginalisation, normalisation and the chain rule of pdfs [12] together with conditional independence expressed by the assumption stated above.

Hence the ith cost to go function for the assumed models of the ith subsystem can be obtained from 4 to yield

$$
\begin{aligned}
& -\ln \left(\gamma\left(w_{t ; i}\right)\right)=\min _{c\left(u_{t ; i} \mid w_{t ; i}\right)} \int s\left(w_{t+1 ; i} \mid u_{t ; i}, w_{t ; i}\right) c\left(u_{t ; i} \mid w_{t ; i}\right) \\
& \times[\underbrace{\ln \left(\frac{s\left(w_{t+1 ; i} \mid u_{t ; i}, w_{t ; i}\right) c\left(u_{t ; i} \mid w_{t ; i}\right)}{I_{s}\left(w_{t+1 ; i} \mid u_{t ; i}, w_{t ; i}\right)^{I} c\left(u_{t ; i} \mid w_{t ; i}\right)}\right)}_{\equiv \text { partial cost } \Longrightarrow \mathrm{U}\left(w_{\mathrm{t}+1 ; i}, u_{t ; i}\right)} \\
& -\underbrace{\ln \left(\gamma\left(w_{t+1 ; i}\right)\right)}_{\text {optimal cost-to-go }}] \mathrm{d}\left(w_{t+1 ; i}, u_{t ; i}\right)
\end{aligned}
$$

Using the ith state model 8 , the ideal distribution of the $i$ th subsystem 9, and the ideal distribution of the ith controller ${ }^{\mathrm{I}} \mathrm{c}\left(\mathrm{u}_{\mathrm{t} ; \mathrm{i}} \mid w_{\mathrm{t} ; \mathrm{i}}\right)$ in 11 and the chain rule yields,

$$
\begin{aligned}
& -\ln \left(\gamma\left(w_{t ; i}\right)\right)=\int s\left(x_{t+1 ; i} \mid u_{t ; i}, w_{t ; i}\right) s\left(y_{t+1 ; i} \mid y_{t ; i}\right) \\
& \times c\left(u_{t ; i} \mid w_{t ; i}\right)\left[\ln \frac{s\left(x_{t+1 ; i} \mid u_{t ; i}, w_{t ; i}\right)}{I_{s}\left(x_{t+1 ; i} \mid u_{t ; i}, w_{t ; i}\right)}\right. \\
& \left.+\ln \frac{c\left(u_{t ; i} \mid w_{t ; i}\right)}{I_{c}\left(u_{t ; i} \mid w_{t ; i}\right)}-\ln \left(\gamma\left(w_{t+1 ; i}\right)\right)\right] d\left(x_{t+1 ; i}, y_{t+1 ; i}, u_{t ; i}\right)
\end{aligned}
$$

Now using Fubini theorem, we introduce the following definitions

$$
\begin{aligned}
& \ln \left(\tilde{\gamma}\left(x_{t+1 ; i}, y_{t ; i}\right)\right)=\int s\left(y_{t+1 ; i} \mid y_{t ; i}\right) \ln \left(\gamma\left(w_{t+1 ; i}\right)\right) d y_{t+1 ; i} \\
& \beta\left(u_{t ; i}, w_{t ; i}\right)=\int s\left(x_{t+1 ; i} \mid u_{t ; i}, w_{t ; i}\right)\left[\ln \frac{s\left(x_{t+1 ; i} \mid u_{t ; i}, w_{t ; i}\right)}{I_{s}\left(x_{t+1 ; i} \mid u_{t ; i}, w_{t ; i}\right)}\right. \\
& \left.-\ln \left(\tilde{\gamma}\left(x_{t+1 ; i}, y_{t ; i}\right)\right)\right] d x_{t+1 ; i} \\
& =\int s\left(x_{t+1 ; i} \mid u_{t ; i}, w_{t ; i}\right) \ln \frac{s\left(x_{t+1 ; i} \mid u_{t ; i}, w_{t ; i}\right)}{I_{s}\left(x_{t+1 ; i} \mid u_{t ; i}, y_{t ; i}\right) \tilde{\gamma}\left(x_{t+1 ; i}, y_{t ; i}\right)} \\
& d x_{t+1 ; i}
\end{aligned}
$$

Substitute 14 and 13 in 12 , we get

$$
\begin{aligned}
& -\ln \left(\gamma\left(w_{t ; i}\right)\right)=\int c\left(u_{t ; i} \mid w_{t ; i}\right)\left[\beta\left(u_{t ; i}, w_{t ; i}\right)\right. \\
& \left.+\ln \frac{\mathrm{c}\left(\mathrm{u}_{\mathrm{t} ; \mathrm{i}} \mid w_{\mathrm{t} ; \mathrm{i}}\right)}{\mathrm{I}_{\mathrm{C}}\left(\mathrm{u}_{\mathrm{t} ; \mathrm{i}} \mid w_{\mathrm{t} ; \mathrm{i}}\right)}\right] \mathrm{d} \mathrm{u}_{\mathrm{t} ; \mathrm{i}} \\
& =\int c\left(u_{t ; i} \mid w_{t ; i}\right) \ln \frac{c\left(u_{t ; i} \mid w_{t ; i}\right)}{I_{c}\left(u_{t ; i} \mid w_{t ; i}\right) \exp \left(-\beta\left(u_{t ; i}, w_{t ; i}\right)\right)} d u_{t ; i} \\
& =\int c\left(u_{t ; i} \mid w_{t ; i}\right)\left[\ln \frac{c\left(u_{t ; i} \mid w_{t ; i}\right)}{\frac{I_{c}\left(u_{t ; i} \mid w_{t ; i}\right) \exp \left(-\beta\left(u_{t ; i}, w_{t ; i}\right)\right)}{\gamma\left(w_{t ; i}\right)}}\right. \\
& \left.-\ln \gamma\left(w_{t ; i}\right)\right] d u_{t ; i}
\end{aligned}
$$

By adding and subtracting $\ln \left(\gamma\left(\mathcal{w}_{\mathrm{t} ; \mathrm{i}}\right)\right)$, the first term in 15 has become a conditional version of the KL divergence. The independence of $\ln \gamma\left(w_{t ; i}\right)$ on the optimised $c\left(u_{t, i} \mid w_{t ; i}\right)$ implies that the expression is minimised by the claimed pdf 10 .

\section{Linear GAUSSIAN QUADRATIC DESIGN}

To demonstrate the distributed control development discussed in the previous section, we consider the regulation problem of a stochastic linear Gaussian state space model described by,

$$
\begin{aligned}
x_{t+1} & =A x_{t}+B u_{t}+\eta_{t+1} \\
s\left(x_{t+1} \mid x_{t}, u_{t}\right) & \rightsquigarrow \mathcal{N}_{x_{t+1}}\left(A x_{t}+B u_{t}, \Sigma\right),
\end{aligned}
$$

where $A$ and $B$ are the state and control matrices respectively, $\eta_{t+1}$ is zero mean white normal innovations, and where $\Sigma$ is the covariance of the innovations. The matrices $A, B$, and $\Sigma>0$ are assumed to be known.

For the considered regulation problem, the system is initially in state $x_{t}$ and the aim is to return the system state to the origin. Before we discuss the distributed control solution for this regulation problem we briefly review the standard solution to the problem using the global FPD control method.

\section{A. Global FPD Control Method}

The first step to obtaining the solution to the standard regulation problem is to identify the ideal distribution of the system states given in equation 16. This ideal state distribution is assumed to be given by,

$$
{ }^{\mathrm{I}} \mathrm{s}\left(\mathrm{x}_{\mathrm{t}+1} \mid \mathrm{u}_{\mathrm{t}}, \mathrm{x}_{\mathrm{t}}\right)=\mathcal{N}_{\mathrm{x}_{\mathrm{t}+1}}(0, \Sigma)
$$

The above definition of the ideal distribution reflects the regulation problem with the realistic aim of reaching the zero 
state, with a spread being determined by the covariance of the innovations, $\Sigma$.

The randomized controller to be designed is described by,

$$
\begin{aligned}
c\left(u_{t} \mid x_{t}\right) & \rightsquigarrow \mathcal{N}_{u_{t}}\left(C x_{t}, \Gamma\right) \\
u_{t} & =C x_{t}+\epsilon_{t},
\end{aligned}
$$

where $C$ is the matrix of the controller parameters, $\epsilon_{t}$ is zero mean white normal innovations, and $\Gamma$ is the covariance of the innovations of control. The distribution of the ideal controller is also assumed to be

$$
{ }^{\mathrm{I}} \mathrm{c}\left(\mathrm{u}_{\mathrm{t}} \mid \mathrm{x}_{\mathrm{t}}\right)=\mathcal{N}_{\mathrm{u}_{\mathrm{t}}}(0, \Gamma),
$$

By using the randomized controller 18, the stochastic equation of the system state 16 can be recast as follows:

$$
x_{t+1}=(A+B C) x_{t}+B \epsilon_{t}+\eta_{t+1} .
$$

Now, if the pair $(A, B)$ is stabilisable, i.e. if there is such a controller $C_{0}$ that makes all eigenvalues of the matrix $A+B C_{o}$ lie inside the unit circle then the optimal randomised controller minimising the cost-to-go function for horizon $H \rightarrow \infty$ is stabilising and has the form [14], [16]

$$
u_{t}=C x_{t}+\epsilon_{t}
$$

with

$$
C=-\left(B^{\top} M B+B^{\top} \Sigma^{-1} B+\Gamma^{-1}\right)^{-1}\left(B^{\top} M A+B^{\top} \Sigma^{-1} A\right),
$$

$$
\begin{aligned}
& M=A^{\top} \Sigma^{-1} A+A^{\top} M A-\left(A^{\top} M B+A^{\top} \Sigma^{-1} B\right) \\
& \left(B^{\top} M B+B^{\top} \Sigma^{-1} B+\Gamma^{-1}\right)^{-1}\left(B^{\top} M A+B^{\top} \Sigma^{-1} A\right),
\end{aligned}
$$

where $M$ is the matrix of the attained quadratic cost function

$$
-\ln \left(\gamma\left(\mathbf{x}_{\mathrm{t}}\right)\right)=0.5 \mathbf{x}_{\mathrm{t}}^{\top} M \mathbf{x}_{\mathrm{t}}+\mathbb{Q}_{0},
$$

and where $\mathbb{Q}_{0} \geq 0$ is some positive constant. The derived solution 23 coincides with stationary algebraic Riccati equation for linear quadratic design [17].

\section{B. Distributed FPD Control Method}

The application of FPD distributed controllers to regulate the system 16 is carried out by designing $|\mathbf{I}|$ randomised controllers described by,

$$
\begin{aligned}
c\left(u_{t ; i} \mid w_{t ; i}\right) & \rightsquigarrow \mathcal{N}_{u_{t ; i}}\left(C_{i} w_{t ; i}, \Gamma_{i}\right) \\
u_{t ; i} & =C_{i} w_{t ; i}+\epsilon_{t ; i},
\end{aligned}
$$

where $i \in \mathbf{I} \equiv\{1, \ldots,|\mathbf{I}|\}$ and where $C_{i}$ is the matrix of the ith controller parameters, $\epsilon_{t ; i}$ is zero mean white normal innovations of the $i$ th control input, and $\Gamma_{i}$ is the covariance of the innovation of ith control. Each of these randomized controllers is responsible for controlling the state values of the corresponding ith subsystem, $x_{t+1 ; i}$ while not influencing the rest of the states $y_{t+1 ; i}$. As such, $|\mathbf{I}|$ models of the $|\mathbf{I}|$ subsystems are required to be identified, one for the derivation of the parameters of each randomized controller. The ith model of the ith subsystem describes the small controlled subpart $x_{t+1 ; i}$ of the state, while models part of the states of its neighbours $y_{t+1 ; i}$ as an external observable disturbance,

$$
\begin{aligned}
& w_{t+1 ; i}=A_{i} w_{t ; i}+B_{i} u_{t ; i}+\eta_{t+1 ; i} \\
& s\left(w_{t+1 ; i} \mid u_{t ; i}, w_{t ; i}\right)=s\left(x_{t+1 ; i} \mid u_{t ; i}, w_{t ; i}\right) s\left(y_{t+1 ; i} \mid y_{t ; i}\right) \\
& \rightsquigarrow \mathcal{N}_{x_{t+1 ; i}}\left(A_{i ; x} w_{t ; i}+B_{i} u_{t ; i}, \Sigma_{i ; x}\right) \\
& \mathcal{N}_{y_{t+1 ; i}}\left(A_{i ; y} y_{t ; i}, \Sigma_{i ; y}\right)
\end{aligned}
$$

Following the discussion in Section II, the ideal distribution of the closed loop model of the ith subsystem takes the pdf modelling the external variables to be equal to the ideal (desirable) pdf of external variables, thus not influencing their values,

$$
\begin{aligned}
& { }^{I} s\left(x_{t+1 ; i} \mid u_{t ; i}, w_{t ; i}\right) s\left(y_{t+1 ; i} \mid y_{t ; i}\right) \\
& \rightsquigarrow \mathcal{N}_{x_{t+1 ; i}}\left(0, \Sigma_{i ; x}\right) \mathcal{N}_{y_{t+1 ; i}}\left(A_{i ; y} y_{t ; i}, \Sigma_{i ; y}\right)
\end{aligned}
$$

The distribution of the ith ideal controller is also assumed to be

$$
{ }^{I} c\left(u_{t ; i} \mid w_{t ; i}\right)=\mathcal{N}_{u_{t ; i}}\left(0, \Gamma_{i}\right),
$$

The considered distributed control let each input entry $u_{t ; i}$ be generated by ith controller working with the ith model, hence yielding the following closed loop response of the controlled ith subpart of the system state,

$$
w_{t+1 ; i}=\left(A_{i}+B_{i} C_{i}\right) w_{t ; i}+B_{i} \epsilon_{t ; i}+\eta_{t+1 ; i}
$$

Similarly here, if the pair $\left(A_{i}, B_{i}\right)$ is stabilisable, i.e. if there is such a controller $C_{i ; o}$ that makes all eigenvalues of the matrix $A_{i}+B_{i} C_{i ; o}$ lie inside the unit circle then the optimal randomised distributed feedback control law minimising the cost-to-go function 10 for horizon $\mathrm{H} \rightarrow \infty$ subject to the pdf of the system state values 26 and ideal distributions of system state 27 and controller 28 is stabilising and has the form

$$
u_{t ; i}=C_{i} w_{t ; i}+\epsilon_{t ; i}
$$

with

$$
\begin{aligned}
& C_{i}=-\left(B_{i}^{\top} M_{i} B_{i}+B_{i}^{\top} \Sigma_{i}^{-1} B_{i}+\Gamma_{i}^{-1}\right)^{-1} \\
& \left(B_{i}^{\top} M_{i} A_{i}+B_{i}^{\top} \Sigma_{i}^{-1} A_{i}\right), \\
& M_{i}=A_{i}^{\top} \Sigma_{i}^{-1} A_{i}+A_{i}^{\top} M_{i} A_{i}-\left(A_{i}^{\top} M_{i} B_{i}\right. \\
& \left.+A_{i}^{\top} \Sigma_{i}^{-1} B_{i}\right)\left(B_{i}^{\top} M_{i} B_{i}+B_{i}^{\top} \Sigma_{i}^{-1} B_{i}+\Gamma_{i}^{-1}\right)^{-1} \\
& \left(B_{i}^{\top} M_{i} A_{i}+B_{i}^{\top} \Sigma_{i}^{-1} A_{i}\right),
\end{aligned}
$$

where $M_{i}$ is the matrix of the obtained quadratic cost function from the $i$ th model, $A_{i}=\left[A_{i ; x} ; A_{i ; y}\right]$, and $\Sigma_{i}$ is given by

$$
\left[\begin{array}{cc}
\Sigma_{i ; x} & 0 \\
0 & 0
\end{array}\right]
$$

which considers the spread around the ith subpart of the state and takes the rest of the state as external variables.

$$
-\ln \left(\gamma\left(w_{t ; i}\right)\right)=0.5 w_{t ; i}^{\top} M_{i} w_{t ; i}+\mathbb{Q}_{0 ; i}
$$

and where $\mathbb{Q}_{0 ; i} \geq 0$ is some positive constant. 
These distributed optimized controllers will then exchange information or messages about the parameters of the dynamics of the subparts of the system and will update these parameters iteratively in a probabilistic framework until they converge to the true values. Specifically, the ith optimized randomized controller offers its neighbour the part of the resulting optimal closed loop related to the variables it perceives as external variables, so that it can be used to improve the description of these external variable. To elaborate the following algorithm can be readily applied to the neighbour distributed controllers and models and can be used for updating the parameters of the dynamics of the various subparts in the system,

- Given the initial state values $\mathcal{w}_{t ; i}$

- For model $i$ estimate the parameters of the $i$ th subpart of the system states, $A_{i ; x}$, and $B_{i, x}$.

- For model $i$ initialize the parameters of the external state variables of the system states, $A_{i ; y}$.

- At each time instant $t$

- Loop

- Use Equation 31 to calculate the feedback gain of the ith controller.

- Optimally tuned closed loop from the view point of the ith controller has the form given in equation 29 , repeated here,

$$
w_{t+1 ; i}=\left(A_{i}+B_{i} C_{i}\right) w_{t ; i}+B_{i} \epsilon_{t ; i}+\eta_{t+1 ; i}
$$

- From 34 we can update the parameters for the jth subpart of the system as follows,

$$
w_{t+1 ; j \leftarrow i} \Rightarrow A_{j ; y} w_{t ; j}=\left(A_{i ; x}+B_{i, x} C_{i}\right) w_{t ; i}
$$

where $w_{t+1 ;(j) \leftarrow(i)}$ is used to denote models of the $j$ th node as obtained from the ith node.

\section{Coupled Map Lattice Network And NUMERICAL RESULTS}

We validate and illustrate the distributed probabilistic control method presented in Section III-B using the stochastic version [14] of a coupled map lattice (CML) with periodic boundary condition as an example of complex dynamical networks which was originally introduced in [18]. The obtained results of the distributed probabilistic controllers will also be compared to the global pinning control solution to the considered CML problem as discussed in section III-A. We first give an overview of the coupled map lattice control problem.

\section{A. Overview of Coupled Map Lattice}

Consider a CML consisting of $\mathrm{L}$ nodes with periodic boundary conditions,

$$
\begin{aligned}
& z_{\mathrm{t}+1}^{j}=\mathrm{F}\left(z_{\mathrm{t}}^{j-1}, z_{\mathrm{t}}^{j}, z_{\mathrm{t}}^{j+1}\right) \\
& =\mathrm{f}\left[(1-2 \epsilon) z_{\mathrm{t}}^{j}+\epsilon\left(z_{\mathrm{t}}^{j-1}+z_{\mathrm{t}}^{j+1}\right)\right]+\kappa_{\mathrm{t}+1}^{j},
\end{aligned}
$$

where $j=1,2, \ldots, L$ are the lattice sites, $L$ is the system size, $\kappa_{t+1}^{j}$ is an additive noise signal assumed to have zero mean Gaussian distribution of covariance $\rho$, and the periodic boundary conditions are given by $z_{t}^{j+L}=z_{t}^{j}$. The local map $f(z)$ is defined to be a nonlinear function of the following form

$$
f(z)=a z(1-z)
$$

This coupled map lattice exhibits chaotic characteristics in the regime $3.57<a \leq 4.0$ and has a homogeneous steady state $z^{\star}=1-1 / \mathrm{a}$.

The objective here is to stabilize the homogeneous state of the lattice by designing a number of controllers that achieve this objective. Because of the complexity and the large number of nodes of the CML, this goal is conventionally achieved via pinning control methodology by applying $\mathrm{K}$ periodically control actions placed at sites $\left\{\mathfrak{j}_{1}, \ldots, j_{K}\right\}$ in the following way

$$
z_{\mathrm{t}+1}^{j}=\mathrm{F}\left(z_{\mathrm{t}}^{\mathrm{j}-1}, z_{\mathrm{t}}^{\mathrm{j}}, z_{\mathrm{t}}^{\mathrm{j}+1}\right)+\sum_{\mathrm{k}=1}^{\mathrm{K}} \delta\left(j-\mathrm{j}_{\mathrm{k}}\right) \mathrm{u}_{\mathrm{t}}^{\mathrm{k}}+\mathrm{k}_{\mathrm{t}+1}^{\mathrm{j}},
$$

where $u_{k}$ is the control action applied at site $k$.

Traditionally in pinning control the theory of linear quadratic control is used to calculate the feedback control actions. for that purpose Equation 36 is linearized about the homogeneous steady state $\mathbf{z}_{\mathrm{t}}=\left(z_{1}^{\star}, \ldots, z_{\mathrm{L}}^{\star}\right)$ to become as follows

$$
x_{t+1}=A x_{t}+B u_{t}+\eta_{t+1}
$$

in which $x=\mathbf{z}-\mathbf{z}^{\star}$ represents the state vector, the $\mathrm{L} \times \mathrm{L}$ Jacobian matrix $A$, is given by

$A=\alpha\left[\begin{array}{ccccc}1-2 \epsilon & \epsilon & 0 & \cdots & \epsilon \\ \epsilon & 1-2 \epsilon & \epsilon & \cdots & 0 \\ 0 & \epsilon & 1-2 \epsilon & \ldots & 0 \\ \vdots & \vdots & \vdots & \ddots & \vdots \\ \epsilon & 0 & 0 & \ldots & 1-2 \epsilon\end{array}\right]$

where $\alpha=\left.\frac{\partial f(z)}{\partial z}\right|_{z=z^{\star}}$, and B is an $L \times M$ control matrix with $B_{j m}=\sum_{k}^{\partial z} \delta(m-k) \delta\left(j-j_{k}\right)$.

Following the discussion in Section III-A the pinning controlled network as a result of the designed randomised controller 21 will have the form given by equation 20 , repeated here

$$
x_{t+1}=(A+B C) x_{t}+B \epsilon_{t}+\eta_{t+1},
$$

where $C$ is calculated as specified by equation 22. In [19], the control matrix, B is chosen such that the network of systems is made controllable as opposed to stabilizable. Since the coupled map lattice considered has parity symmetry [19], the eigenvalues of its Jacobian are doubly degenerate. Therefore, the minimal number of controllers that yields a controllable system in this case is two which will be assumed in the current paper.

In contrast to the pinning control method, the proposed distributed control method considers designing and optimizing $|\mathbf{I}|$ number of controllers, each is responsible for controlling and regulating a predefined collective number of neighbouring nodes in the lattice. For the linearized version of CML 39, the distributed controllers can be designed 
as discussed in Section III-B. The ith subsystem of the controlled network as a result of the ith controller will then have the form given by equation 34 , repeated here

$$
w_{t+1 ; i}=\left(A_{i}+B_{i} C_{i}\right) w_{t ; i}+B_{i} \epsilon_{t ; i}+\eta_{t+1 ; i}
$$

where $C_{i}$ is calculated as specified by equation 31 .

\section{B. Numerical Results of Global FPD}

The example considered here is for the logistic coupled map lattice, $f(z)=a z(1-z)$ in its non-chaotic regime with $a=3.0, \epsilon=0.33$ and $L=5$ and with an external Gaussian random input, $\eta_{t+1}$ affecting the dynamics. Following the pinning control method [14], two controllers are used and they are placed next to each other at the sides of the lattice. Hence the equation of the coupled map lattice becomes:

$$
x_{t+1}=A x_{t}+B u_{t}+\eta_{t+1}
$$

where

$$
\begin{gathered}
A=\left[\begin{array}{ccccc}
-0.34 & -0.33 & 0 & 0 & -0.33 \\
-0.33 & -0.34 & -0.33 & 0 & 0 \\
0 & -0.33 & -0.34 & -0.33 & 0 \\
0 & 0 & -0.33 & -0.34 & -0.33 \\
-0.33 & 0 & 0 & -0.33 & -0.34
\end{array}\right], \\
B=\left[\begin{array}{ll}
1 & 0 \\
0 & 0 \\
0 & 0 \\
0 & 0 \\
0 & 1
\end{array}\right] \quad \Sigma=E\left[\eta_{t+1} \eta_{t+1}^{\top}\right]=0.001 \mathrm{I}_{5 \times 5} .
\end{gathered}
$$

The lattice is initially at time $t=0$ in state $x=0.25$ and the aim is to return the lattice to the origin (the fixed point position) or a state close to the origin. The matrices $A$ and $B$ and the covariance matrix $\Sigma>0$ are assumed to be known. The covariance matrix of the controller is taken to be $\Gamma=$ $0.1 \mathrm{I}_{2 \times 2}$. The resulted states of the lattice network and the obtained control efforts are illustrated in Figures 1(a) and (b), respectively, which show that the controlled network is globally synchronized by the designed global probabilistic pinning controller. These obtained results will be compared to the distributed control results which will be discussed in the following section.

\section{Numerical Results of Distributed FPD Control}

For comparison purposes, the same logistic coupled map lattice, $f(z)=a z(1-z)$ in its non-chaotic regime with $a=3.0, \epsilon=0.33$ and $L=5$ and with an external Gaussian random input, $\eta_{t+1}$ affecting the dynamics will be considered here for the design of distributed probabilistic controller. We design two distributed probabilistic controllers $u_{t ; 1}$ and $u_{t ; 2}$. The first probabilistic controller $u_{t ; 1}$ is concerned with the regulation of the first three nodes in the lattice while modelling the rest of the states as an external observable disturbance,

$$
w_{t+1 ; 1}=A_{1} w_{t, 1}+B_{1} u_{t ; 1}+\eta_{t+1 ; 1}
$$

where

$$
\begin{aligned}
& A_{1}=\left[\begin{array}{ccccc}
-0.34 & -0.33 & 0 & 0 & -0.33 \\
-0.33 & -0.34 & -0.33 & 0 & 0 \\
0 & -0.33 & -0.34 & -0.33 & 0 \\
0 & 0 & 0 & a_{1 ; 44} & 0 \\
0 & 0 & 0 & 0 & a_{1 ; 55}
\end{array}\right], \\
& B_{1}=[1,1,1,0,0]^{\top} \\
& \text { and } \Sigma_{1}=E\left[\eta_{t+1 ; 1} \eta_{t+1 ; 1}^{\top}\right]=\left[\begin{array}{cc}
0.001 \mathrm{I}_{3 \times 3} & 03 \times 2 \\
0_{2 \times 3} & 0_{2 \times 2}
\end{array}\right],
\end{aligned}
$$

The second probabilistic controller $u_{t ; 2}$ is concerned with the regulation of the last three nodes in the lattice while modelling the rest of the states as an external observable disturbance,

$$
w_{\mathrm{t}+1 ; 2}=A_{2} w_{t ; 2}+B_{2} u_{t ; 2}+\eta_{t+1 ; 2},
$$

where

$$
\begin{aligned}
A_{2} & =\left[\begin{array}{ccccc}
a_{2 ; 11} & 0 & 0 & 0 & 0 \\
0 & a_{2 ; 22} & 0 & 0 & 0 \\
0 & -0.33 & -0.34 & -0.33 & 0 \\
0 & 0 & -0.33 & -0.34 & -0.33 \\
-0.33 & 0 & 0 & -0.33 & -0.34
\end{array}\right], \\
B_{2} & =[0,0,1,1,1]^{\top} \\
\Sigma_{2} & =E\left[\eta_{t+1 ; 2} \eta_{t+1 ; 2}^{\top}\right]=\left[\begin{array}{cc}
0_{2 \times 2} & 0 \\
0_{3 \times 2} & 0.001 I_{3 \times 3}
\end{array}\right] .
\end{aligned}
$$

The four unknown parameters $a_{1 ; 44}, a_{1 ; 55}, a_{2 ; 11}$, and $a_{2 ; 22}$ are related to the external observable states from the point of view of the first and second controllers and are to be initialized randomly. The two controllers then exchange information about the closed loop response of the system which is used to update these unknown parameters as discussed in Section III-B.

Similarly here for comparison purpose, the lattice is assumed to be initially at time $t=0$ in state $x=0.25$ and the aim is to return the lattice to the origin (the fixed point position) or a state close to the origin. The covariance matrices of the first and second controllers are taken to be $\Gamma_{1}=0.1, \Gamma_{2}=0.1$ respectively. The resulted states of the lattice network and the obtained control efforts are illustrated in Figures 1(c) and (d), respectively. The results from these figures show that the proposed distributed probabilistic controller, is very effective in globally regulating the controlled CML network.

\section{Conclusion}

The problem of controlling complex dynamical systems with large number of nodes has been considered in this paper. the proposed solution is developed via distributed stochastic control where noise and uncertainties are contained using probabilistic approaches. This solution considers a bottom up strategy where simple controllers are designed to care about individual subparts of the system either completely independently or within various structures like cascade control. By utilising the FPD approach, we developed the general methodology for distributed control proposed in this paper. 
CONFIDENTIAL. Limited circulation. For review only.

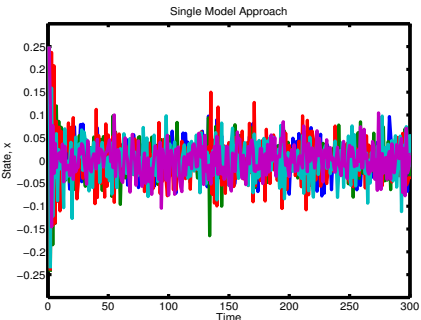

(a)

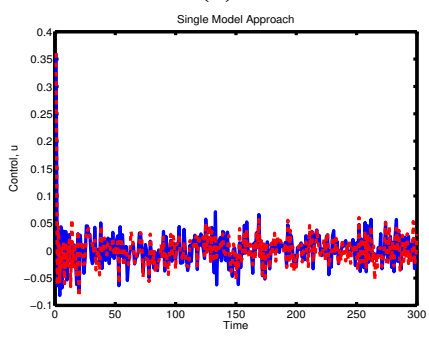

(b)

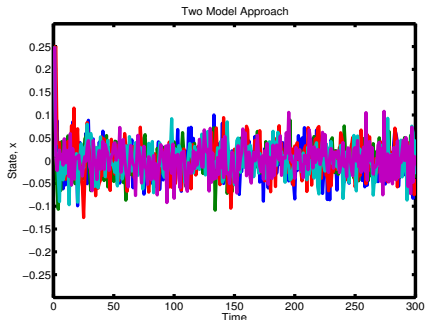

(c)

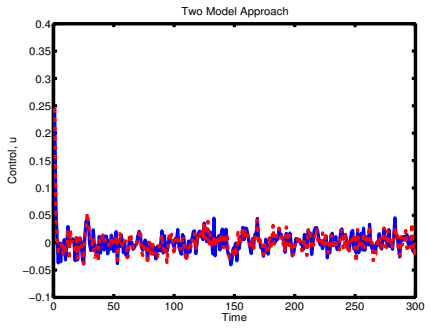

(d)
Fig. 1. States and control efforts of a non chaotic coupled map lattice with, $L=5, a=3$, and $\epsilon=0.33$ : (a) states as a result of the global FPD controller. (b) control efforts from the global controller. (c) states as a result of the distributed FPD controller. (d) control efforts from the distributed controller.

The developed distributed controllers exchange information about the various nodes in the network and use this information to update their governing dynamical relations. The theoretical findings were then validated on a coupled map lattice network as an example of complex dynamical networks. Numerical results confirm the effectiveness of the proposed distributed probabilistic control approach in globally regulating the states of the network.

\section{REFERENCES}

[1] M. Porfiri and M. di Bernardo, "Criteria for global pinning controllability of complex networks," Automatica, vol. 44, pp. 3100-3106, 2008.

[2] B. V. den. Broek, W. Wiegerinck, and B. kappen, "Graphical model inference in optimal control of stochastic multi-agent systems," Journal of Artifial Intelligence Research, vol. 33, pp. 95-122, 2008.

[3] D. D. Śiljak and A. I. Zečević, "Control of large scale systems: Beyond decentralised feedback," Annual Reviews in Control, vol. 29, pp. 169$179,2005$.

[4] Z. Wang, R. Lu, and B. Shen, "Distributed estimation and control for general systems," International Journal of General Systems, vol. 43, no. 3-4, pp. 247-253, 2014.

[5] L. Zdeborová, A. Decelle, and M. Chertkov, "Message passing for optimization and control of a power grid: Model of a distribution system with redundancy," Phys. Rev. E, vol. 80, p. 046112, Oct 2009. [Online]. Available: http://link.aps.org/doi/10.1103/PhysRevE.80.046112

[6] S. M. Aji and R. J. McEliece, "The generalized distributed law," IEEE Transactions on Information Theory, vol. 46, no. 2, pp. 325-343, 2000.

[7] R. G. Gallager, Low-Density Parity Check Codes. M.I.T. Press, Cambridge, MA, 1963.

[8] J. Pearl, Probabilistic Reasoning in Intelligent Systems. Morgan Kaufman, San Mateo, CA, 1988.

[9] G. P. M. Mézard and M. A. Virasoro, Spin Glass Theory and Beyond. World Scientific, Singapore, 1987.

[10] M. Kárńy, "Towards fully probabilistic control design," Automatica, vol. 32, no. 12, pp. 1719-1722, 1996.

[11] R. Herzallah and M. Kárný, "Fully probabilistic control design in an adaptive critic framework," Neural Networks, vol. 24, no. 4, pp. 11281135, 2011.
[12] V. Peterka, "Bayesian system identification," in Trends and Progress in System Identification, P. Eykhoff, Ed. Oxford: Pergamon Press, 1981, pp. 239-304.

[13] R. Herzallah, "Probabilistic dhp adaptive critic for nonlinear stochastic control systems," Neural Networks, vol. 42, pp. 74-82, June 2013.

[14] - "Probabilistic synchronisation of pinning control," International Journal of Control, pp. 1-9, July 2012, DOI:10.1080/00207179.2012.700488.

[15] C. Rao, Linear method of statistical inference and their applications. Prague: Academia, 1987, in Czech.

[16] T. V. Guy and M. Kárný, "Stationary fully probabilistic control design," in Proceedings of the Second International Conference on Informatics in Control, Automation and Robotics, J. Filipe, J. A. Cetto, and J. L. Ferrier, Eds. Barcelona: INSTICC, September 2005, pp. $109-112$.

[17] J. Meditch, Stochastic Optimal Linear Estimation and Control. Mc. Graw Hill, 1969.

[18] H. Gang and Q. Zhilin, "Controlling spatiotemporal chaos in coupled map lattice systems," Physical Review Letters, vol. 72, no. 1, pp. 6873, January 1994.

[19] R. O. Grigoriev, M. C. Cross, and H. G. Schuster, "Pinning control of spatiotemporal chaos," Physical Review Letters, vol. 79, no. 15, pp. 2795-2798, October 1997. 